\title{
Polarimetric \& Spectropolarimetric Properties of FGK Type Stars and Extrasolar Systems
}

\author{
S. Ortolani ${ }^{\dagger}$, F. Tamburini ${ }^{\ddagger} \&$ A. Bianchini ${ }^{\dagger}$ \\ $\dagger$ Università di Padova, Dipartimento di Astronomia, Vicolo \\ dell'Osservatorio 2, I-35122 Padova, Italy. \\ emails: bianchini@pd.astro.it,ortolani@pd.astro.it \\ $\ddagger$ Institute of Cosmology and Gravitation, \\ Univ. of Portsmouth, PO2 1EG, Portsmouth, UK \\ email: fabrizio.tamburini@port.ac.uk
}

\section{Introduction}

The study of extra solar planets represents a new exciting frontier for modern astronomy. Most of the results on extrasolar systems are obtained with Doppler surveys. Here we suggest a different approach aimed to study the polarimetric properties of the residual interplanetary dust. Dust can produce observable effects such as infrared emission excess (Beckwith \& Sargent 1996; Spangler et al. 2001) and partial polarization of the star light via scattering (Mauron \& Dole 1998; Yudin 2000), like in $\beta$ Pictoris (Voshchinnikov \& Krügel 1999). Some polarization could also be produced by a giant planet in a close orbit around the star (Saeger et al. 2000), but the resulting effect is too weak to be responsible for the polarizations actually observed.

\section{Polarization properties}

The polarization data are taken from the Heiles catalogue (Heiles 2000) by selecting stars within a distance of $70 p c$, that roughly corresponds to the largest distance amongst the observed extrasolar planet systems so far observed. The main characteristics of the extra-solar planetary systems within our sample are given in the Planet Encyclopedia (Schneider 2002). The errors in the polarization measurements are those reported in Heiles catalogue, typically around $0.03 \%$.

We have compared the polarization distribution of extrasolar systems to that of a selected sample of stars without planets ${ }^{1}$. The two distributions shown in Fig. 1 are different, as confirmed by the Kolmogorov-Smirnov test with $99 \%$ confidence level. Single stars show a maximum between $p=0.01-0.02$, while stars with planets show a well defined maximum around 0 and a rather steeper decline towards higher polarization degrees.

\footnotetext{
${ }^{1}$ Since we do not observe extrasolar systems with $p>0.09$, we have taken this value as an upper limit also for the sample of single stars.
} 
Plotting in Fig. 2 the orbital eccentricities of the extrasolar planets $v s$ their polarization, we find a peculiar distribution of the data points, since only the lower left part of the diagram is populated, while the upper right region is almost empty. Highly eccentric orbits only have low polarization levels, while high polarizations are detected only in low eccentricity systems. In any case the polarization levels of many of the stars with planetary systems in the figure appear surprisingly higher than predicted by current models of scattering from circumstellar dust.
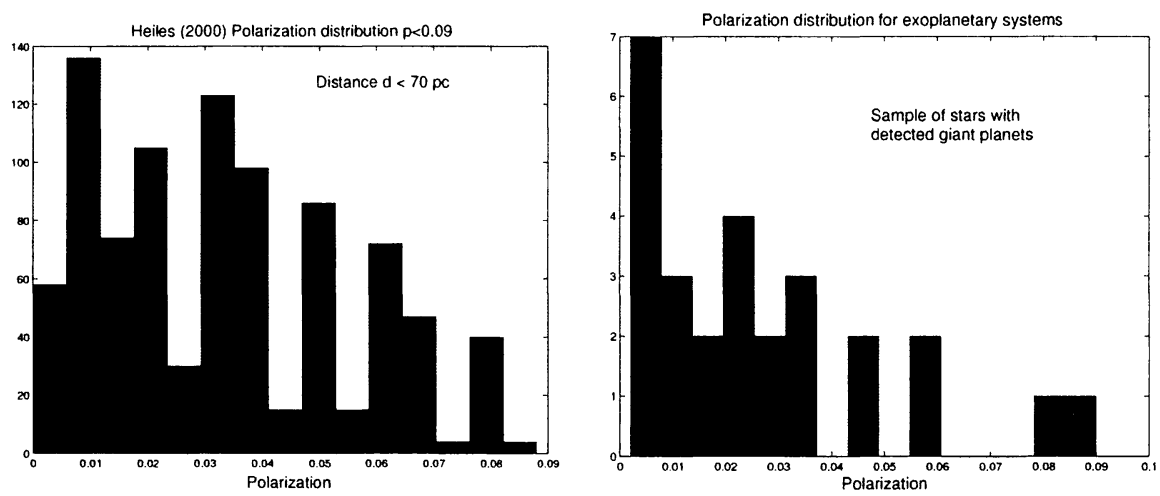

Figure 1. (left panel) Histogram of the distribution of the polarization within $70 p c$ and $\mathrm{Pol}<0.09$ of stars from Heiles Catalogue within $70 p c$ and $\mathrm{Pol}<0.09$. (right panel) Polarization distribution of the sample of stars with detected giant planets. A well defined peak around $p \sim 0$ is evident.

Given the surprising result, we investigated other possible effects (Tamburini et al. 2002). Distance and age seem to be irrelevant for the observed distribution. Considering that the mass of the circumstellar dust disks have been suggested to be a function of the age (Spangler et al. 2001), we checked if the higher polarization of HD 179949 and Tau Bootis could be due to a younger age. HD 179949 is 3.31 Gyr old, and Tau Bootis is 2.52 Gyr (Chen 2001; Marsakov 1995), while, for example Epsilon Eridani, which has a rather low polarization $(p=0.01)$, is instead rather younger (about $1 \mathrm{Gyr}$ ). We note that the only exception, the F9V star HD 114762, which has $p=0.09$ and $e=0.334$, has a closeby substellar object companion; an object closer to a brown dwarf candidate (Latham et al. 1989) rather than a planet.

At present it is not clear what mechanism could be responsible for the observed polarization levels and orbital eccentricities in our sample. We have started systematic spectropolarimetric observations at the Asiago Observatory in order to check with a higher accuracy, and for a wider sample, these effects. Preliminary results show that the polarization accuracy we can achieve is of the order of $\sim 0.01 \%$ and the low polarization of the high eccentricity systems seems confirmed. 


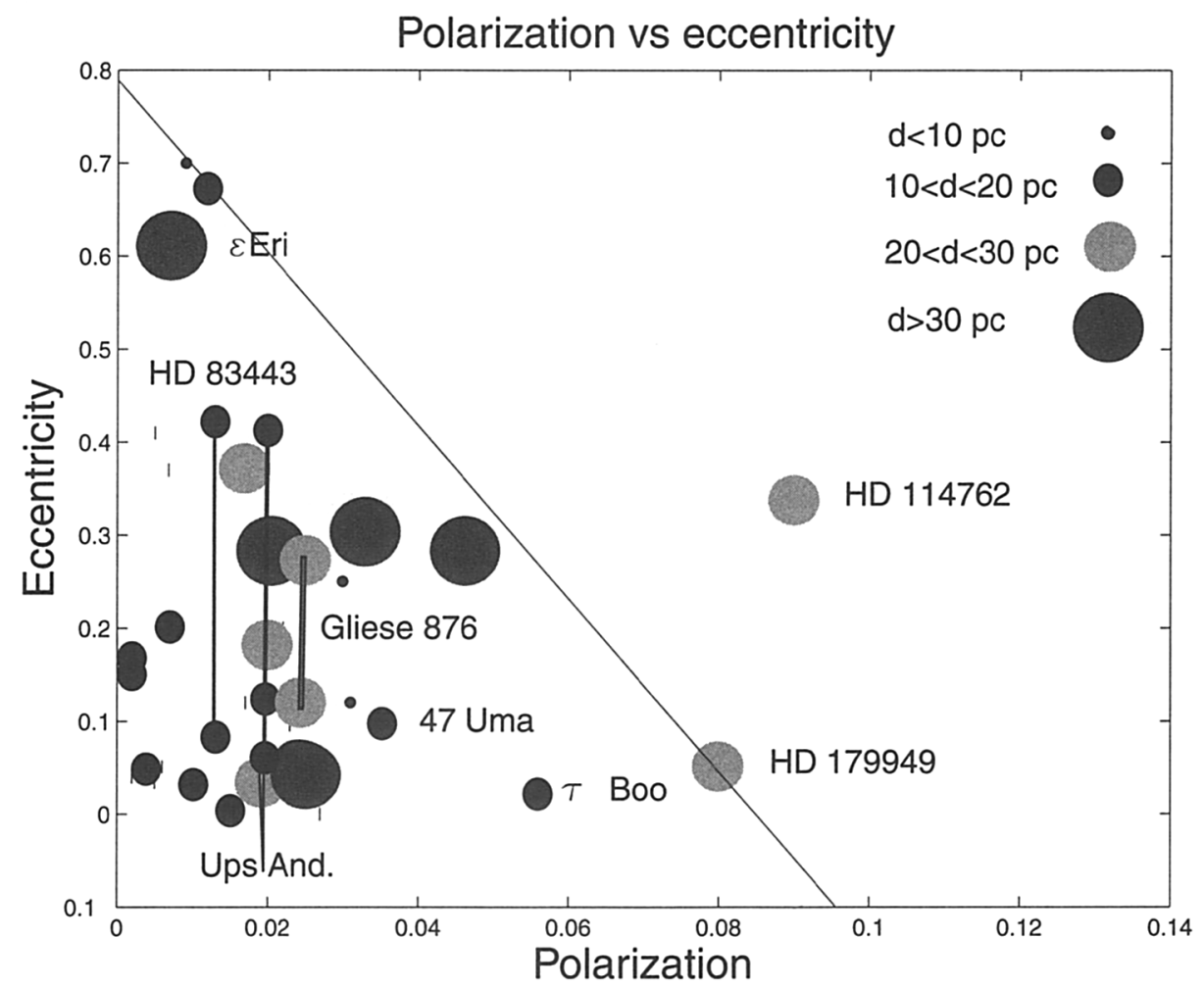

Figure 2. Polarization $v s$ orbital eccentricity. The extrasolar systems are mainly distributed in the lower left region of the diagram. The diagonal line tentatively separates the two regions in the diagram. The exception of HD 114762 is discussed in the text. There is indication that larger polarizations are allowed at lower eccentricities. Multiple systems are connected by dotted lines. Symbol sizes correspond to different distances: no distance effects can be seen.

\section{Conclusions}

We have analyzed the statistical polarimetric properties of extrasolar systems taken from the Heiles catalogue (Heiles 2000). We have compared the polarization distribution of extrasolar systems with that of a selected sample of stars with no planet detected. The most significant result found is that only systems with low eccentricity orbits can have high polarization values. We have also found that at distances $<70 p c$ there is no relevant contribution due to interstellar dust. We have started an observational spectropolarimetric program to analyse the circumstellar material. 


\section{References}

Beckwith, S. V. W., \& Sargent, A. I. 1996, Nature, 383, 139

Chen, Y. Q., Nissen, P. E., Benoni, T., Zhao, G. 2001, A\&A, 371, 943

Heiles, C. 2000, AJ, 119, 923

Latham, D. W., Mazeh, T., Stefanik, R. P., Mayor, M., \& Burki, G. 1989, Nature, 339,38

Marsakov, V. A., Shevelev, Yu. G. 1995, Bull. Inf. Centre Donnees Stellaires, (Bull. Inf. CDS), 47, 13

Mauron N., Dole H. 1998, A\&A, 337, 808

Saeger, S. Whitney, B. A., \& Sasselov, D. D. 2000, ApJ, 540, 504

Spangler, C., Sargent, A. I., Silverstone, M. D., Becklin, E. E., \& Zuckerman, B. 2001, ApJ, 555, 932

Schneider, J. 2002, in Extrasolar Planets Encyclopedia, http://www.obspm.fr/planets

Tamburini, F., Ortolani, S., \& Bianchini, A. 2002, A\&A, 394, 675

Voshchinnikov, N. V., \& Krügel, E. 1999, A\&A, 352, 508

Yudin, R. V. 2000, A\&AS, 144, 285

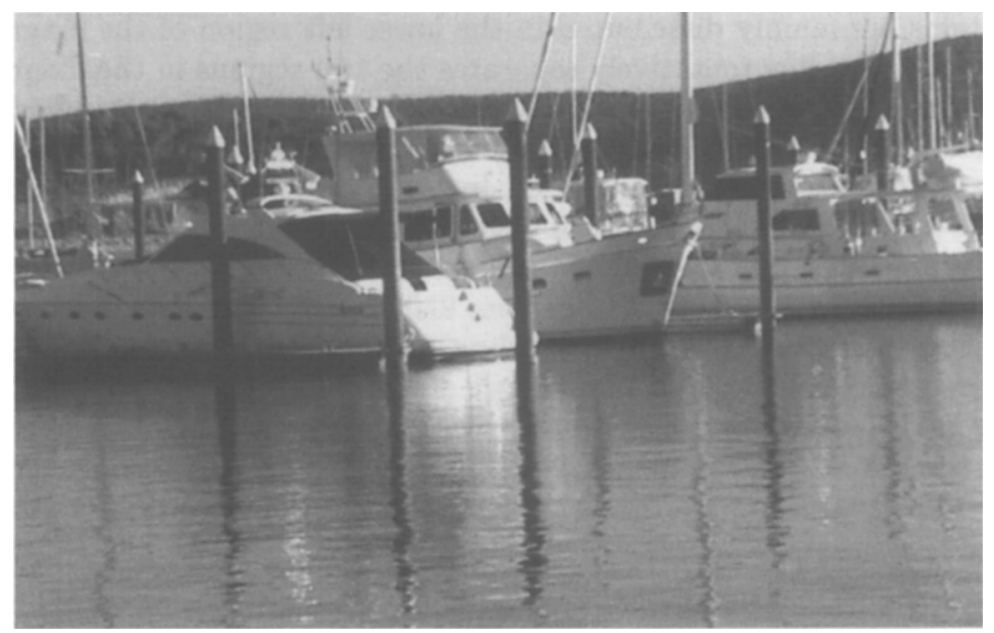

(photo: Daniella Scalise) 